\title{
Find them early
}

Getting young researchers into independent positions that encourage risk-taking would benefit science but requires more than targeted individual research grants.

The public perception is that important scientific advances are made by scientists with at least a few gray hairs, but the reality is that most scientists perform their groundbreaking work early in their careers. In fact, a 1993 study of Nobel Prize recipients stated, “....although it does not require extraordinary youth to do prizewinning work, the odds decrease markedly in mid-life and fall off precipitously after age $50 . . . "$

Whether it is their willingness to take greater risks, a better capacity to think 'outside the box' or the enthusiasm and energy of youth, it is clearly in the interests of science to ensure that the most promising young scientists be provided with fully supported independent positions that foster risky innovative research.

Distressingly, the median age of US National Institutes of Health (NIH) grantees has risen steadily from about 33 in 1970 to nearly 50. Many initiatives have been proposed to stem this dangerous trend. Five years ago the National Research Council released the "Bridges to Independence" report, calling for changes in funding and support by 2010 in an effort to promote effective independence for early career investigators. In response, the NIH established the Pathway to Independence and New Innovator awards and in 2009 it stated that investigators applying for RO1 grants within 10 years of their terminal degree would be given special consideration.

These initiatives involve evaluation of specific research proposals. But if the goal is to identify individuals with the greatest potential to perform innovative research, such evaluation can be ineffective. It is more appropriate to evaluate the individual rather than a proposal.

Such a model can be successful. When the European Molecular Biology Laboratory (EMBL) was created in the 1970s, the organizers saw a dynamic, highly successful research culture in the US driven by the rapid ascent of graduates into independent positions. In contrast with the stratified research environment that dominated Europe — where senior researchers were ensconced in stable positions and upward mobility for talented researchers was frustratingly slow-US researchers often received their first $\mathrm{RO} 1$ grant in their twenties.

EMBL is structured so group leaders are recruited at an early stage in their career - either upon graduating or from an early postdoc — and limited to a 9-year non- renewable term, after which they can return to their home country as independent researchers. Organizers hoped this would instill youthful vigor to European research. Selection is based on stringent personal evaluations, and the most promising candidates are often identified by tracking people through personal contacts. The goal is to find people with good ideas and ambitious plans. Appointments can occur even in the face of poor publication records. Judged by quality of research, the model is a success. According to citation numbers, EMBL has the highest impact of any European research institute in the two 10 -year periods since 1990.

When the Howard Hughes Medical Institute established the Janelia Farm Research Campus, EMBL was one of their models. Both Janelia and the National Institute of Biological Sciences in Beijing have been aggressive at hiring early career investigators into their 5 -year positions. The success of this model relies on careful candidate selection and sufficient support to quickly establish a research program, including multiple lab personnel and strong core facilities. Management classes can also help, but quality mentoring from senior colleagues is more valuable.

Senior colleagues are also a source for collaborations. Limiting lab sizes makes collaboration essential and dissuades empire-building efforts that may foster big science at the expense of innovative science.

Recruiting quality personnel is a challenging and crucial part of starting a lab. At EMBL, PhD candidates and postdocs are interviewed by multiple group leaders, and new group leaders are given priority in choosing who to hire after obtaining input from colleagues. The presence of a small proportion of established labs is considered essential for attracting quality postdocs.

More institutes using this model would help ensure that the individuals with the most to offer science have an opportunity to do so before the vagaries of funding and employment drive them to something perceived to be more stable. Early stage career candidates are also gender balanced. Providing the resources to be successful early enough, along with reasonable maternity leave and onsite child care, may help redress the gender imbalance among senior scientist role models and mentors and start a virtuous cycle for rising female scientists and stem the loss of talented prospects. 